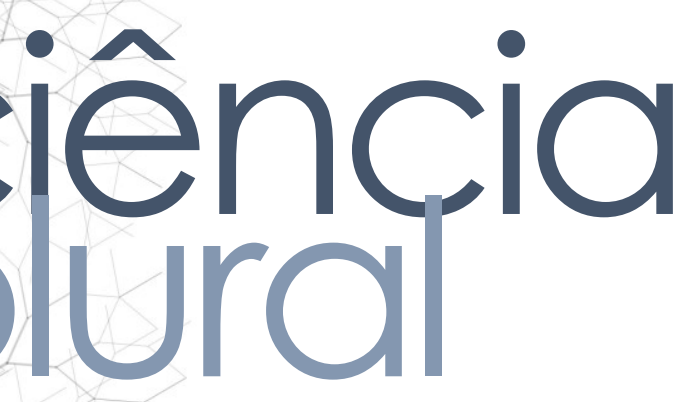

\title{
EFICÁCIA DE ÓLEOS VEGETAIS NO CONTROLE DO BIOFILME DENTAL: REVISÃO INTEGRATIVA
}

Efficacy of vegetable oils in control of dental biofilm: integrative review

Eficacia de los aceites vegetales en el control de biofilm dental: revision integrativa

Suila Gaia de Oliveira • Cirurgiã Dentista graduada pela Faculdade de Odontologia da Universidade Federal do Pará • E-mail: suilagaiatrabalho@hotmail.com

Danielle Tupinambá Emmi • Professora da Faculdade de Odontologia da Universidade Federal do Pará • E-mail: dtemmi@ufpa.br

Autora correspondente:

Danielle Tupinambá Emmi • E-mail: dtemmi@ufpa.br 


\section{ciêncíncia
puúl}

\section{RESUMO}

Introdução: A terapia de bochecho com óleos vegetais é uma cultura tradicional na Índia com intuito de prevenir afecções bucais, como cárie e doença periodontal. Atualmente, com as mudanças nos padrões alimentares da sociedade, tem-se empregado com maior valor o uso de óleos naturais, já que estudos demonstram sua importância para saúde. Objetivo: Esta revisão integrativa teve como objetivo selecionar evidências que mostrassem a utilização de óleos vegetais, com ação antimicrobiana, no controle do biofilme dental. Metodologia: O levantamento bibliográfico foi realizado nos meses de julho e agosto de 2020, por meio de pesquisa por via eletrônica, consultando-se o banco de dados Pubmed e SciELO (Scientific Electronic Library Online). Foram utilizados os descritores: Óleos vegetais, agentes antimicrobianos, Placa dental, Odontologia preventiva e Odontologia, os descritores foram usados separadamente e em cruzamento com o operador boleano AND. Utilizou-se os seguintes critérios de inclusão: Estudos clínicos, artigos completos e disponíveis gratuitamente, publicados no período de 2010 a 2020 em português e/ou inglês, e indexados nas bases de dados já citadas. Foram excluídos os trabalhos de conclusão de curso, dissertações, teses, livros e capítulos de livros, além de artigos duplicados e que não abordaram o tema do estudo. Resultados: Atenderam os critérios da pesquisa 04 estudos do tipo clínico randomizado, os quais mostraram eficiência dos óleos vegetais utilizados no controle do biofilme dental. A realização de pesquisas envolvendo e motivando a industrialização de produtos naturais contribui para a Política Nacional de Práticas Integrativas e Complementares. Conclusões: Os artigos analisados mostraram eficácia na utilização de óleos vegetais no controle do biofilme dental, sendo esses aplicados clinicamente, não mostrando prejuízo aos participantes e indicados como coadjuvante na manutenção da saúde bucal.

Palavras-Chave: Óleos vegetais; Agentes antimicrobianos; Placa dental; Odontologia preventiva; Odontologia.

\section{ABSTRACT}

Introduction: Mouthwash therapy with vegetable oils is a traditional culture in India with the aim of preventing oral diseases, such as caries and periodontal disease. Currently, with the changes in the dietary patterns of society, the use of natural oils has been used with greater value, since studies demonstrate its importance for health. Objective: This integrative review aimed to select evidence that showed the use of vegetable oils, with antimicrobial action, in the control of dental biofilm. Methodology: The bibliographic survey was carried out in July and August 2020, through electronic research, consulting the Pubmed and SciELO database (Scientific Electronic Library Online). The descriptors were used: Vegetable oils, antimicrobial gents, dental plaque, preventive dentistry and dentistry, the descriptors were used eparately and in cross-section with the Boolean operator AND. The following clusion criteria were used: Clinical studies, complete and freely available articles, blished between 2010 and 2020 in Portuguese and / or English, and indexed in the tabases already cited. Course completion papers, dissertations, theses, books and k chapters were excluded, as well as duplicate articles that did not address the 
subject of the study. Results: Four studies of randomized clinical type met the research criteria, which showed efficiency of vegetable oils used in the control of dental biofilm. Conducting research involving and motivating the industrialization of natural products contributes to the National Policy on Integrative and Complementary Practices. Conclusions: The analyzed articles showed efficacy in the use of vegetable oils in the control of dental biofilm, which were applied clinically without showing any harm to the participants and indicated as an adjunct in maintaining oral health.

Keywords: Plant oils; Anti-infective agents; Dental plaque; Preventive dentistry; Dentistry.

\section{RESUMEN}

Introducción: La terapia de enjuague bucal con aceites vegetales es una cultura tradicional en India con el objetivo de prevenir enfermedades bucales, como caries y enfermedad periodontal. Actualmente, con los cambios en los patrones dietéticos de la sociedad, se ha utilizado con mayor valor el uso de aceites naturales, ya que los estudios demuestran su importancia para la salud. Objetivo: Esta revisión integradora tuvo como objetivo seleccionar evidencias que mostraran el uso de aceites vegetales, con acción antimicrobiana, en el control del biofilm dental. Metodología: El relevamiento bibliográfico se realizó en julio y agosto de 2020, mediante investigación electrónica, consultando la base de datos Pubmed y SciELO (Scientific Electronic Library Online). Se utilizaron los descriptores: Aceites vegetales, agentes antimicrobianos, placa dental, odontología preventiva y odontología, los descriptores se utilizaron por separado y en corte transversal con el operador booleano AND. Se utilizaron los siguientes criterios de inclusión: Estudios clínicos, artículos completos y de libre acceso, publicados entre 2010 y 2020 en portugués y / o inglés, e indexados en las bases de datos ya citadas. Se excluyeron trabajos de finalización de curso, disertaciones, tesis, libros y capítulos de libros, así como artículos duplicados que no abordaran el tema de estudio. Resultados: Cuatro estudios de tipo clínico aleatorizado cumplieron con los criterios de investigación, los cuales mostraron la eficiencia de los aceites vegetales utilizados en el control del biofilm dental. Realizar investigaciones que involucren y motiven la industrialización de productos naturales contribuye a la Política Nacional de Prácticas Integrativas y Complementarias. Conclusiones: Los artículos analizados mostraron eficacia en el uso de aceites vegetales en el control del biofilm dental, los cuales fueron aplicados clínicamente sin mostrar daño a los participantes e indicados como coadyuvante en el mantenimiento de la salud bucal.

alabras clave: Aceites vegetales; Agentes antimicrobianos; Placa dental; Odontología preventiva; Odontología. 


\section{Introdução}

O conjunto formado pelos seres vivos e os elementos ambientais é representado pelos microrganismos e o habitat, sendo que as condições ambientais das diferentes regiões do nosso organismo selecionam e determinam as espécies capazes de colonizar, crescer e tornar-se membro de uma comunidade residente, determinandose ecossistema. Apesar da microbiota bucal parecer um sistema de crescimento aberto, significando que os nutrientes e os microrganismos são introduzidos e removidos repetidamente, a capacidade de aderência às superfícies da cavidade bucal, faz com que espécies se estabeleçam compondo uma variedade de ecossistemas distintos, por isso, a microbiota da boca é dividida em quatro nichos principais, o biofilme dental, sulco gengival, dorso da língua e mucosas ${ }^{1}$.

O biofilme dental, um dos principais nichos de comunidade microbiana, é o fator etiológico da cárie e da doença periodontal. A prevalência dessas afecções sintetiza que ainda há um desafio por melhorias na prevenção dos problemas bucais, devendo-se, dessa forma, enfatizar o controle do biofilme dental².

Os lipídios em suas características parecem ser promissores no meio odontológico, já que influenciam na interação microbiana, dificultando a agregação de microrganismos, por formar uma camada hidrofóbica na superfície dental diminuindo a susceptibilidade à cárie³ .

A possibilidade de remoção do biofilme dental usando um creme dental experimental a base de óleo de amêndoas, foi testada por Aguiar e Saliba ${ }^{4}$, que observaram que os óleos vegetais seriam mais eficientes na manutenção da saúde oral e provocariam menos abrasão.

Os enxágues bucais com óleos, usados na Índia por muitos anos como terapia tradicional para limpar os dentes, gengivas, eliminar toxinas da boca e prevenir cáries, não eram sustentadas por evidências ${ }^{5}$. Entretanto, estudos recentes utilizando dessa terapia com os óleos de girassol, de gergelim e de côco reduziram a formação de biofilme como também a gengivite induzida por ela ${ }^{6}$.

Os óleos podem ser obtidos de plantas oleaginosas ou sementes, e vem sendo lizado de maneira diversificada em muitas culturas ao longo dos séculos. Suas 
aplicações incluem aditivos alimentares, novas tecnologias, produtos para saúde e aplicação na indústria de $\operatorname{cosméticos}^{3}$.

Estudo in situ realizado com os óleos extraídos da polpa dos frutos de Astrocaryum vulgare (tucumã) e Bactris gasipae (pupunha) identificou o potencial antimicrobiano dos referidos óleos na redução da agregação bacteriana do biofilme dental 7 .

Considerando que os lipídeos são capazes de alterar a composição da película e assim reduzir a agregação bacteriana na formação do biofilme, o objetivo dessa revisão foi reunir estudos que utilizaram óleos vegetais, com aplicação antimicrobiana, para o controle do biofilme dental.

\section{Metodologia}

Para construção deste estudo, foi realizada uma revisão integrativa da literatura, que se constitui como um método que proporciona a síntese de conhecimento da aplicabilidade de resultados de estudos significativos na prática ${ }^{8}$. A pesquisa seguiu as 06 fases de elaboração de revisões sistemáticas: elaboração da pergunta norteadora, busca ou amostragem na literatura, coleta de dados, análise crítica dos estudos incluídos, discussão dos resultados e apresentação da revisão integrativa9.

A pergunta norteadora para a elaboração da revisão integrativa foi: Quais as evidências de pesquisas que avaliam a eficácia de óleos vegetais no controle de biofilmes dentais?

O levantamento bibliográfico foi realizado nos meses de julho e agosto de 2020, por meio de pesquisa por via eletrônica, consultando-se o banco de dados Pubmed e SciELO (Scientific Electronic Library Online).

A busca ou amostragem da literatura foi realizada de acordo com os Descritores em Ciências da Saúde (DeCS): Óleos vegetais (Plant oils), agentes ntimicrobianos (Anti-Infective Agents), Placa dental (Dental plaque), Odontologia reventiva (Preventive Dentistry), Odontologia (Dentistry). Sendo esses usados aradamente e em cruzamento com o operador boleano AND. 
Para extrair os dados dos artigos selecionados, se fez a utilização de um instrumento validado por $\mathrm{Ursi}^{10}$ capaz de assegurar que os dados relevantes fossem extraídos, minimizando o risco de erros na transcrição, garantindo precisão na checagem das informações e servindo como registro.

Os critérios de inclusão definidos para a seleção dos artigos foram: Estudos clínicos, artigos completos e disponíveis gratuitamente, publicados no período de 2010 a 2020 em português e/ou inglês, e indexados nas bases de dados já citadas. Foram excluídos os trabalhos de conclusão de curso, dissertações, teses, livros e capítulos de livros, além de artigos duplicados e que não abordaram o tema do estudo ${ }^{10}$

Figura 1: Fluxograma referente a seleção de artigos para este estudo. Belém-PA, 2020.

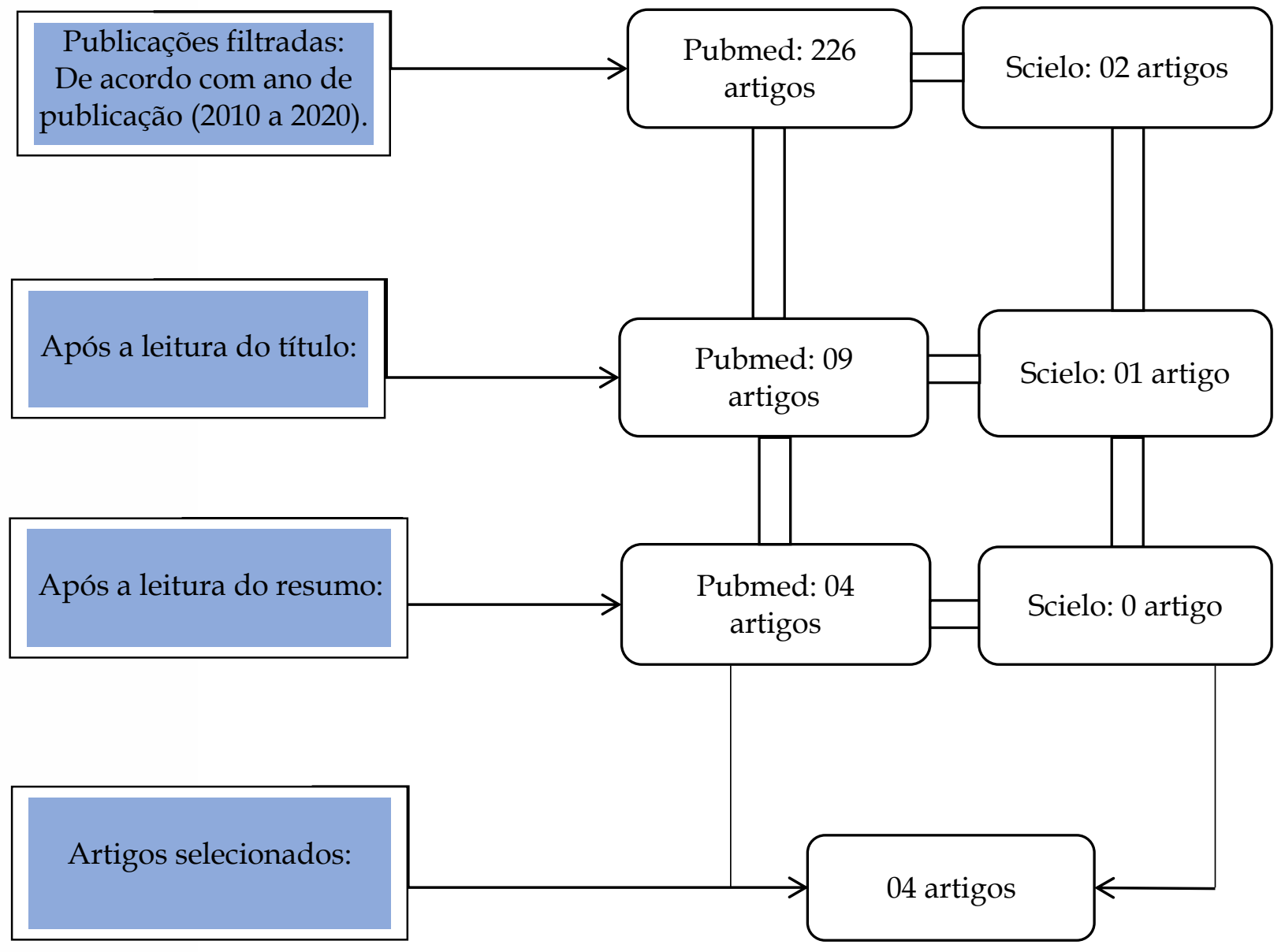




\section{Resultados}

Foram previamente selecionados 10 artigos após a leitura do título, 09 na base de dados Pubmed e 01 na base Scielo. Após a leitura do resumo, 04 artigos atenderam os critérios de inclusão, e 06 artigos foram descartados, sendo 05 artigos da Pubmed e 01 da Scielo. Os artigos excluídos não atenderam aos critérios de inclusão da pesquisa.

Quadro 1: Apresentação dos artigos incluído na revisão sobre a eficácia de óleos vegetais no controle de biofilmes dentais. Belém-PA, 2020.

\begin{tabular}{|c|c|c|c|}
\hline $\begin{array}{c}\text { TÍTULO/ } \\
\text { ANO }\end{array}$ & OBJETIVO & $\begin{array}{c}\text { PRINCIPAIS } \\
\text { RESULTADOS }\end{array}$ & CONCLUSÃO \\
\hline $\begin{array}{l}\text { Effect of vegetable } \\
\text { oil (Brazil nut oil) } \\
\text { and mineral oil } \\
\text { (liquid petrolatum) } \\
\text { on dental biofilm } \\
\text { control } \\
\text { Filogônio et al., } \\
\text { 2011. }\end{array}$ & $\begin{array}{l}\text { Avaliar } \\
\text { a eficácia da } \\
\text { adição de óleo } \\
\text { vegetal ou } \\
\text { mineral a um } \\
\text { dentifrício } \\
\text { comercialmente } \\
\text { disponível no } \\
\text { controle de } \\
\text { biofilme dental. }\end{array}$ & $\begin{array}{l}\text { Uma comparação usando } \\
\text { o índice de Higiene Oral } \\
\text { Simplificado (IHO-S) foi } \\
\text { realizada em } 30 \\
\text { indivíduos que foram } \\
\text { divididos aleatoriamente } \\
\text { em três grupos. } \\
\text { O grupo } 1 \text { (G1) recebeu } \\
\text { um dentifrício } \\
\text { comercialmente } \\
\text { disponível; a composição } \\
\text { desse dentifrício foi } \\
\text { modificada pela adição } \\
\text { de óleo mineral (Nujol) } \\
\text { para o grupo } 2 \text { (G2) ou } \\
\text { de um vegetal } \\
\text { (Alpha Care) para o } \\
\text { grupo } 3 \text { (G3) a } 10 \% \text { do } \\
\text { volume total, } \\
\text { respectivamente. } \\
\text { A análise revelou uma } \\
\text { redução significativa no } \\
\text { OHI-S no dia } 90 \text { no G2 } \\
\text { (p <0,05) e G3 (p <0,0001) } \\
\text { em comparação ao G1. }\end{array}$ & $\begin{array}{l}\text { A adição } \\
\text { de um óleo vegetal ou } \\
\text { mineral a um } \\
\text { dentifrício } \\
\text { comercialmente } \\
\text { disponível } \\
\text { melhorou o controle } \\
\text { do biofilme dental, } \\
\text { sugerindo que esses } \\
\text { óleos podem auxiliar } \\
\text { na prevenção e/ou } \\
\text { controle de cárie e } \\
\text { doença periodontal. }\end{array}$ \\
\hline
\end{tabular}




\begin{tabular}{|c|c|c|c|}
\hline $\begin{array}{l}\text { Effect of sesame oil, } \\
\text { ozonated sesame } \\
\text { oil, } \\
\text { and chlorhexidine } \\
\text { mouthwash on oral } \\
\text { health } \\
\text { status of } \\
\text { adolescents: A } \\
\text { randomized } \\
\text { controlled } \\
\text { pilot trial } \\
\text { Vadhana et al., } \\
2019 \text {. }\end{array}$ & $\begin{array}{l}\text { Avaliar a } \\
\text { eficácia do óleo } \\
\text { de gergelim } \\
\text { (SO), } \\
\text { SO ozonizado } \\
\text { (OSO) e } \\
\text { enxaguatório } \\
\text { bucal de } \\
\text { clorexidina } \\
\text { (CHX) } \\
\text { sobre o estado } \\
\text { de saúde bucal } \\
\text { de adolescentes. }\end{array}$ & $\begin{array}{l}\text { Três grupos (n = 25): } \\
\text { Grupo I (SO), Grupo II } \\
\text { (OSO) e Grupo III } \\
\text { (enxaguatório bucal } \\
\text { CHX). Índice (DI-S), } \\
\text { Índice de Cálculo (CI-S), } \\
\text { Índice de Higiene Oral } \\
\text { simplificado (OHI-S), } \\
\text { índice de placa (PI) e } \\
\text { a contagem salivar de } \\
\text { Streptococcus mutans } \\
\text { foram registrados. } \\
\text { Todos os grupos } \\
\text { apresentaram redução } \\
\text { estatisticamente } \\
\text { significante } \\
\text { em DI - S, CI - S, OHI - S, } \\
\text { PI e Streptococcus mutans } \\
\text { após } 15 \text { dias. }\end{array}$ & $\begin{array}{l}\text { Terapia de bochecho } \\
\text { com óleo usando SO } \\
\text { e OSO mostraram } \\
\text { uma melhora } \\
\text { significativa na } \\
\text { higiene oral. }\end{array}$ \\
\hline $\begin{array}{l}\text { Comparative } \\
\text { Evaluation of } \\
\text { Antiplaque Efficacy } \\
\text { of Coconut Oil } \\
\text { Pulling and a } \\
\text { Placebo, Among } \\
\text { Dental College } \\
\text { Students: A } \\
\text { Randomized } \\
\text { Controlled Trial } \\
\text { Nagilla et al., } 2017\end{array}$ & $\begin{array}{l}\text { Comparar e } \\
\text { avaliar a } \\
\text { eficácia } \\
\text { antiplaca do } \\
\text { óleo de coco } \\
\text { com um } \\
\text { placebo entre } \\
\text { estudantes de } \\
\text { odontologia, na } \\
\text { cidade de } \\
\text { Hyderabad, na } \\
\text { Índia. }\end{array}$ & $\begin{array}{l}\text { Os escores médios de } \\
\text { placa mostraram uma } \\
\text { diferença significativa } \\
\text { no início, no terceiro e } \\
\text { no sétimo dia entre os } \\
\text { grupos de estudo (p } \\
<0,001 \text { ) e controle ( } \mathrm{p} \\
<0,001 \text { ). A comparação } \\
\text { entre os grupos revelou, } \\
\text { embora os escores } \\
\text { médios de placa } \\
\text { tenham sido baixos no } \\
\text { grupo de estudo no } \\
\text { terceiro e no sétimo dia } \\
\text { na comparação com o } \\
\text { grupo controle, mas } \\
\text { houve diferença } \\
\text { significativa apenas no } \\
\text { sétimo dia. Além disso, } \\
\text { a redução média do } \\
\text { percentual dos escores } \\
\text { de placa também foi } \\
\text { significativa apenas no } \\
\text { sétimo dia, com uma } \\
\text { alta redução média de } \\
\text { placa entre os grupos } \\
\text { de estudo (p <0,001). }\end{array}$ & $\begin{array}{l}\text { O óleo de côco é } \\
\text { eficaz no controle dos } \\
\text { níveis de placa. }\end{array}$ \\
\hline
\end{tabular}




\begin{tabular}{|l|l|l|l|}
\hline $\begin{array}{l}\text { Efficacy of oil } \\
\text { pulling therapy } \\
\text { with coconut oil on } \\
\text { four-day } \\
\text { supragingival } \\
\text { plaque growth: A } \\
\text { randomized } \\
\text { crossover clinical } \\
\text { trial }\end{array}$ & $\begin{array}{l}\text { Avaliar os } \\
\text { efeitos } \\
\text { inibidores do } \\
\text { óleo usando um } \\
\text { modelo de } \\
\text { crescimento de } \\
\text { placa de 4 dias } \\
\text { comparado com } \\
\text { 0,2\% de } \\
\text { gluconato de } \\
\text { clorexidina } \\
\text { (CHX). }\end{array}$ & $\begin{array}{l}\text { A terapia de bochecho } \\
\text { com óleo de côco } \\
\text { apresentou atividade } \\
\text { inibitória semelhante a } \\
\text { CHX. }\end{array}$ & $\begin{array}{l}\text { O óleo de coco parece } \\
\text { ter atividade de } \\
\text { inibição de placa } \\
\text { semelhante à da CHX. } \\
\text { Além disso, } \\
\text { causou menos } \\
\text { manchas nos dentes } \\
\text { que o CHX. Esses } \\
\text { achados sugerem que } \\
\text { a terapia de bochehco } \\
\text { com óleo de côco } \\
\text { pode ser uma } \\
\text { alternativa a CHX. }\end{array}$ \\
\hline
\end{tabular}

As buscas para esta pesquisa tiveram como resultado 04 estudos do tipo clínico randomizado, publicado no período de 2010 a 2020, os quais mostraram eficiência dos óleos vegetais utilizados no controle do biofilme dental. A realização de pesquisas envolvendo e motivando a industrialização de produtos naturais contribui para a Política Nacional de Práticas Integrativas e Complementares (PNPIC), que inclui a Fitoterapia como opção preventiva e terapêutica no Brasil11.

Filogônio et al. ${ }^{12}$, ao estudar a eficácia do óleo vegetal oriundo da Bertholletia excelsa (castanha-do-Pará) adicionou-o em um dentifrício disponível no mercado, e comparou seu efeito a outros dentifrícios sem o óleo no controle de biofilme dental, e obtiveram resultados significativos na redução do quantitativo de placa.

Vadhana et al. ${ }^{13}$, avaliaram a eficácia do óleo de gergelim como enxaguatório bucal, e comparando os resultados à clorexidina, todo os grupos mostraram redução no índice de placa, podendo ser empregado como alternativa a CHX.

Nagilla et al. ${ }^{14}$, em um estudo clínico randomizado, avaliaram o efeito antiplaca do óleo de côco em estudantes de odontologia na Índia, tendo como resultado positivo a eficácia no controle da placa.

Sezgin et al. ${ }^{15}$ também avaliaram a atividade clínica do óleo de côco como terapia no controle da placa dental, que apresentou ação inibitória semelhante no crescimento do biofilme em comparação a clorexidina. 


\section{Discussão}

Métodos para o controle do biofilme dental têm sido estudados para limitar os efeitos e a progressão das afecções bucais mais comuns, a cárie e a doença periodontal. Marsh ${ }^{16}$ menciona que o biofilme se acumula em sítio de estagnação, justamente para se proteger contra a remoção. E ainda ressalta que a compreensão desse ecossistema, possibilita o emprego de novos meios de controle. Nesse sentindo, as pesquisas com produtos naturais no meio odontológico têm aumentado nos últimos anos.

O óleo de Castanha-do-Pará (Bertholletia excelsa) quando adicionado a um dentifrício comercial, comparado a outros dois produtos sem o óleo vegetal, após 90 dias de uso, mostrou que os grupos que fizeram a higienização com os cremes dentais com óleo, obtiveram controle do biofilme dental ${ }^{12}$. Esses resultados podem ser suportados pela composição da Castanha-do-Pará, conforme demonstrado por Pena Muniz et al. ${ }^{1817}$, que relatam que a amêndoa da Castanha-do-Pará consiste em 60-70\% de gordura, 15-20\% de proteína, vitaminas lipossolúveis (A, E) e minerais (Ca, Fe, Zn, $\mathrm{Na}, \mathrm{K}$ e Se). E na caracterização do óleo de B. excelsa (castanha-do-Pará), mostra-se fonte de ácidos graxos poli-insaturados, onde predominam ácido oleico 38,5\% e ácido linoleico 31,26\%, representando 70,19\% dos ácidos graxos totais. Dessa forma, pode-se sugerir que possivelmente, os lipídeos influenciam na interação microbiana, dificultando a agregação de microrganismos ${ }^{3}$.

Também utilizando óleos oriundos da Amazônia, Carvalho ${ }^{19} 18$ desenvolveu um estudo in vitro com enxaguantes bucais a base de óleos de tucumã (Astrocaryum vulgare) e pupunha (Bactris gasipae) e comparou a enxaguantes utilizados no mercado a base de clorexidina, triclosan e cloreto de cetilpiridínio, frente a cepas de Aggregatibacter actinomycetemcomitans (ATCC 29522), Lactobacillus fermentum (ATCC 9338), Streptococcus mutans (ATCC 25175), Streptococcus sanguinis (ATCC 10557) e obteve como resultado que o enxaguante desenvolvido com os óleos vegetais apresentou eficácia na ação antimicrobiana contra a maioria dos microrganismos testados, com exceção do Streptococcus sanguinis, possibilitando assim, aplicabilidade dentro da odontologia. 
Os óleos de tucumã e da pupunha apresentam rica composição de ácidos graxos, principalmente de ácido oleico ${ }^{7}$, o que corrobora a resultados de estudos anteriores que mostram a interferência dos óleos vegetais na inibição ou controle da agregação microbiana do biofilme dental. Segundo Osawa et al. ${ }^{19}$, esses ácidos graxos foram encontrados na semente de cacau como principais constituintes ativos, agindo como bactericida sobre Streptococcus mutans.

Um estudo recente, usando o bochecho com o óleo de gergelim em comparação à clorexidina, mostrou resultados estatísticos semelhantes na redução do biofilme dental ${ }^{13}$. O gergelim é uma planta oleaginosa de grande potencial econômico e suas sementes contêm cerca de 50\% de óleo. Rico em ácidos graxos insaturados, como oleico $(45,3 \%)$ e linoleico $(37,7 \%)$, apresenta em sua composição importantes antioxidantes, como o sesamol, a sesamina e a sesamolina ${ }^{20}$. O resultado deste estudo ${ }^{13}$ mostra a relevância considerável dos óleos vegetais no controle do biofilme, como alternativa à clorexidina, devido esta quando utilizada de forma permanente, causar efeitos indesejáveis, como indução da resistência de algumas bactérias, irritações no paladar e uma mudança na flora oral ${ }^{21}$.

O mesmo método do bochecho com o óleo de gergelim, foi testado por Asokan et al. ${ }^{5}$, para avaliar a redução de Streptococcus mutans, microrganismo importante para cariogenicidade do biofilme. Nesse estudo, o biofilme e a saliva dos participantes foram coletados para análise, e a redução na contagem de $S$. mutans foi estatisticamente significativa, evidenciando que alimentos gordurosos são capazes de reduzir o número de bactérias do biofilme dental 6 .

Nagilla et al. ${ }^{14}$ e Sezgin et al. ${ }^{15}$ analisaram o efeito do óleo de côco na redução do biofilme dental, que demonstrou eficácia, com ação inibitória no crescimento do biofilme. Além disso, quando comparado à clorexidina, o óleo de côco causou menos manchamento dental, sugerindo essa terapia como alternativa para a manutenção a saúde bucal.

O óleo de côco contém grande quantidade de lipídeos de baixo peso molecular como o ácido láurico, possui mais ácidos graxos saturados do que insaturados, mas nesse último, predomina o ácido oleico e linoleico. Outros componentes encontrados em menores concentrações estão monoglicerídeos, diglicerídeos, fosfatídeos, 
carotenóides, esteróis como os tocoferóis, que inibem a oxidação, dando resistência oxidativa a esse óleo ${ }^{22}$.

Peedikayil et al. ${ }^{6}$ conduziram um estudo in vivo para determinar a eficácia antimicrobiana do óleo de côco comparado a clorexidina, evidenciando que o óleo de côco foi tão eficaz quanto a clorexidina na redução de Streptococcus mutans.

Entretanto, Hanning et al. ${ }^{2}$ avaliaram os efeitos dos bochechos com os óleos de cártamo, oliva e linhaça na formação inicial do biofilme, e não obtiveram resultados significativos. Jauhari et al. ${ }^{23}$ constataram que o bochecho com óleo mineral não forneceu nenhum benefício adicional como um agente antimicrobiano eficaz na redução da colonização bacteriana. Todavia, os resultados dos óleos vegetais relatados nos ensaios clínicos coletados nesta revisão, mostraram efeitos no controle do biofilme dental, podendo ser consideradas alternativas estratégicas para prevenção à cárie.

Neste contexto, o Brasil, sobretudo a Amazônia, possui espécies promissoras na produção de óleos comestíveis ${ }^{24}$. Dessa forma, a utilização dos óleos vegetais como alternativa na higiene oral possui grande possibilidade de aceitação pela população local.

\section{Conclusões}

Diante dos estudos encontrados na literatura e selecionados para esta revisão foi possível constatar o papel dos óleos vegetais como estratégia para redução da agregação microbiana do biofilme, inclusive em substituição à clorexidina, considerado padrão ouro para o controle de microrganismos bucais. Sugere-se que este papel protetor desempenhado pelos óleos seja em decorrência da rica composição de ácidos graxos. Assim, evidencia-se nos óleos vegetais importante terapia alternativa para a manutenção da saúde bucal. 


\section{Referências}

1. Jorge AOC. Microbiologia e Imunologia Oral. - Rio de Janeiro: Elsevier. 2012. p. 228-300.

2. Hannig C, Kirsch J, Al-Ahmad A, Kensche A, Hannig M, Kümmerer K. Do edible oils reduce bacterial colonization of enamel in situ? Clin Oral Investig. 2013; 17(2):649-658.

3. Kensche A, Reich M, Kümmerer K, Hannig M, Hannig C. Lipids in preventive dentistry. Clin Oral Investig. 2013; 17(3):669-685.

4. Aguiar AAA, Saliba NA. Toothebrushing with vegetable oil: a clinical and laboratorial analysis. Braz Oral Res. 2004; 18(2): 168-73.

5. Asokan S, Rathan J, Muthu MS, et al. Effect of oil pulling on Streptococcus mutans count in plaque and saliva using Dentocult SM Strip mutans test: a randomized, controlled, triple-blind study. J Indian Soc Pedod Prev Dent. 2008; 26(1):12-17.

6. Peedikayil FC, Sreenivasan P, Narayanan A. Effect of coconut oil in plaque related gingivitis - A preliminary report. Niger Med J. 2015; 56(2):143-147.

7. Emmi DT. Influência dos óleos do tucumã (Astrocaryum vulgare) e da pupunha (Bactris gasipae) na composição do biofilme dental e dinâmica do processo de cárie em esmalte: um estudo in situ [Tese]. Universidade de São Paulo; 2013.

8. Souza MT, Silva MD, Carvalho R. Integrative review: what is it? How to do it? Einstein (São Paulo). 2010; 8(1): 102-106.

9. Silva JMD, Verçosa BMG, Nobre FC, de Melo Azevedo L, Silva MLT, Belo ZS, et al. Utilização de fitoterápicos na Odontologia: revisão integrativa. Res Soc Dev. 2020; 9(8): e209985370

10. Ursi ES. Prevenção de lesões de pele no perioperatório: revisão integrativa da literatura. [Dissertação]. Ribeirão Preto: Universidade de São Paulo, Escola de Enfermagem de Ribeirão Preto; 2005.

11. Francisco KSF. Fitoterapia: uma opção para tratamento odontológico. Revista Saúde 2010; 4(1):18-24.

2. Filogônio CFB, Soares RV, Horta MCR, Penido CVSR, Cruz RA. Effect of vegetable oil (Brazil nut oil) and mineral oil (liquid petrolatum) on dental biofilm control. Braz Oral Res 2011; 25(6): 556-561. 
13. Vadhana VC, Sharath A, Geethapriya PR, Vijayasankari V. Effect of sesame oil, ozonated sesame oil, and chlorhexidine mouthwash on oral health status of adolescents: A randomized controlled pilot trial. J Indian Soc Pedod Prev Dent. 2019; 37(4):365-371.

14. Nagilla J, Kulkarni S, Madupu PR, Doshi D, Bandari SR, Srilatha A. Comparative Evaluation of Antiplaque Efficacy of Coconut Oil Pulling and a Placebo, Among Dental College Students: A Randomized Controlled Trial. J Clin Diagn Res. 2017;11(9):ZC08-ZC11.

15. Sezgin Y, Memis Ozgul B, Alptekin NO. Efficacy of oil pulling therapy with coconut oil on four-day supragingival plaque growth: A randomized crossover clinical trial. Complement Ther Med. 2019; 47:102193.

16. Marsh PD. Dental plaque as a microbial biofilm. Caries Res. 2004; 38(3):204-211.

17. Pena Muniz MA, Ferreira Dos Santos MN, da Costa CE, et al. Physicochemical characterization, fatty acid composition, and thermal analysis of Bertholletia excelsa HBK oil. Pharmacogn Mag. 2015;11(41):147-151.

18. Carvalho LJ. Eficácia antimicrobiana de enxaguantes em bactérias bucais. 2018. 22f. Trabalho de Conclusão de Curso [Graduação]- Faculdade de Odontologia, Instituto de Ciências da Saúde, Universidade Federal do Pará, Belém, 2018.

19. Osawa K, Miyazaki K, Shimura S, Okuda J, Matsumoto M, Ooshima T. Identification of cariostatic substances in the cacao bean husk: their antiglucosyltransferase and antibacterial activities. J Dent Res. 2001; 80(11):2000-2004.

20. Arriel NHC, Vieira DJ, Firmino PT. Situação atual e perspectivas da cultura do gergelim no Brasil. In: Queiroz MA, Goedert CO, Ramos SRR (Ed.) Recursos genéticos e melhoramento de plantas para o Nordeste brasileiro. Petrolina: Embrapa Semiárido; Brasilia, DF, 1999.

21. Deng DM, ten Cate JM, Crielaard W. The adaptive response of Streptococcus mutans towards oral care products: involvement of the ClpP serine protease. Eur J Oral Sci. 2010; 115: 363-370.

22. Moretto E, Fett R. Definição de óleos e Gorduras tecnologia de óleos e gorduras vegetais na indústria de alimentos. São Paulo. 1998. 
23. Jauhari D, Srivastava N, Rana V, Chandna P. Comparative Evaluation of the Effects of Fluoride Mouthrinse, Herbal Mouthrinse and Oil Pulling on the Caries Activity and Streptococcus mutans Count using Oratest and Dentocult SM Strip Mutans Kit. Int J Clin Pediatr Dent 2015; 8(2):114-118.

24. Almeida SSD, Rocha Filho GND, Zoghbi MDGB. O potencial da flora oleífera na Amazônia. In: Pesce C. Oleaginosas da Amazônia. 2.ed., rev. Atual. Belém: Museu Paraense Emilio Goeldi; Núcleo de Estudos Agrários e Desenvolvimento Rural, 2009. p. 23-29. 